\title{
The Effects of Different Combinations of Anesthetic Drug on Cardiopulmonary Functions and Recovery Behavior in Horses
}

\author{
Soontaree PetchdeE ${ }^{*}$, Aree Laikul ${ }^{1}$, Juthamas Leklub ${ }^{2}$, Jutamat Rattanakunuprakarn ${ }^{2}$
}

${ }^{1}$ Department of Large Animal and Wildlife Clinical Sciences, Faculty of Veterinary Medicine, Kasetsart University, Kamphaeng Saen Campus 73140, Thailand; ' ${ }^{2}$ KasetsartUniversity Veterinary Teaching Hospital Kamphaeng Saen campus, Faculty of Veterinary Medicine, Kasetsart University, Kamphaeng Saen Campus, 73140, Thailand.

\begin{abstract}
The objective of this study was to identify the effects of combined anesthetic drugs on heart rate variability (HRV), respiratory function and recovery behavior in horses. Eight healthy Thai native crossbred horses were enrolled in this study. Horses were premedicated and induction with 2 protocols, protocol 1; acepromazine $(0.04 \mathrm{mg} /$ $\mathrm{kg})+$ xylazine $(1 \mathrm{mg} / \mathrm{kg})+\mathrm{ketamine}(2.2 \mathrm{mg} / \mathrm{kg})$ and protocol 2 ; detomidine $(5 \mathrm{mcg} / \mathrm{kg})+\mathrm{ketamine}(2.2 \mathrm{mg} / \mathrm{kg})$. Heart rate and heart rate variability of 8 horses were measured 20 minutes before and after administered the anesthetic drugs. Doppler was used to obtained systolic, diastolic and mean blood pressures. After anesthetic drug administration, horses premedicated and induction with acepromazine, xylazine and ketamine had the lower HR values when compared with another protocols. The HRV low frequency (LF) and high frequency (HF) powers ratio increased in horses premedicated and intubation with detomidine and xylazine. The marked hypoventilation were observed after anesthetic drugs administration in both groups. There were only minimal differences in the quality of recovery. However, overall of recovery scores were higher in group of protocol 1. This study demonstrated that acepromazine + xylazine + ketamine preserved the cardiovascular function and safe to use for the surgical applicability in horses.
\end{abstract}

Keywords | Anesthesia, Horse, Autonomic nervous activity, Heart rate variability, Echocardiography

Editor | Muhammad Abubakar, National Veterinary Laboratories, Islamabad, Pakistan.

Received | September 04, 2018; Accepted | October 03, 2018; Published | October 17, 2018

*Correspondence | Soontaree Petchdee, Department of Large Animal and Wildlife Clinical Sciences, Faculty of Veterinary Medicine, Kasetsart University, Kamphaeng Saen Campus 73140, Thailand; Email: fvetstr@ku.ac.th

Citation | Petchdee S, Laikul A, Leklub J, Rattanakunuprakarn J (2018). The effects of different combinations of anesthetic drug on cardiopulmonary functions and recovery behavior in horses. Res. J. Vet. Pract. 6(3): 20-25.

DOI | http://dx.doi.org/10.17582/journal.rjvp/2018/6.3.20.25

ISSN (Online) | 2308-2798

Copyright (C) 2018 Petchdee et al. This is an open access article distributed under the Creative Commons Attribution License, which permits unrestricted use, distribution, and reproduction in any medium, provided the original work is properly cited.

\section{INTRODUCTION}

$\mathrm{M}$ orbidity and mortality in anesthesia of horses are considerably greater and more pronounced complications than any other species (Johnston et al., 2002). Many anesthetic protocols have been developed to improve the outcomes and provide the safety for anesthesia in horses (Carregaro et al., 2007; Love et al., 2013). Alpha agonist such as detomidine and xylazine is a long-acting sedative that is commonly used for anesthesia in horses, disadvantages of detomidine include ataxia, bradycardia, arrhythmias and reduction in cardiac output (Yamashita et al., 2000). Previous studies reported that alpha adrenergic receptor agonists combination with opioid drug provid- ed the synergistic analgesic effects in horses (Marly et al., 2014; Oliveira et al., 2014). Ketamine is commonly used for induction in horses, combination of an alpha agonist with ketamine provided a smooth induction with reliable sedation and recovery. However, the addition of muscle relaxant such as diazepam can be used to balance the side effects of muscle rigidity from ketamine (Jarrett et al., 2018). Heart rate variability (HRV) has been suggested to measure as a noninvasive index for autonomic nervous control (Pagani et al., 1986; Kishi, 2012). Many studies have been reported the response of heart rate variability to many effects on medications and stress in human and many animal species (Karez et al., 2003; Kato et al., 2003). HRV measurements have been used as a predictor of mortality in pa- 


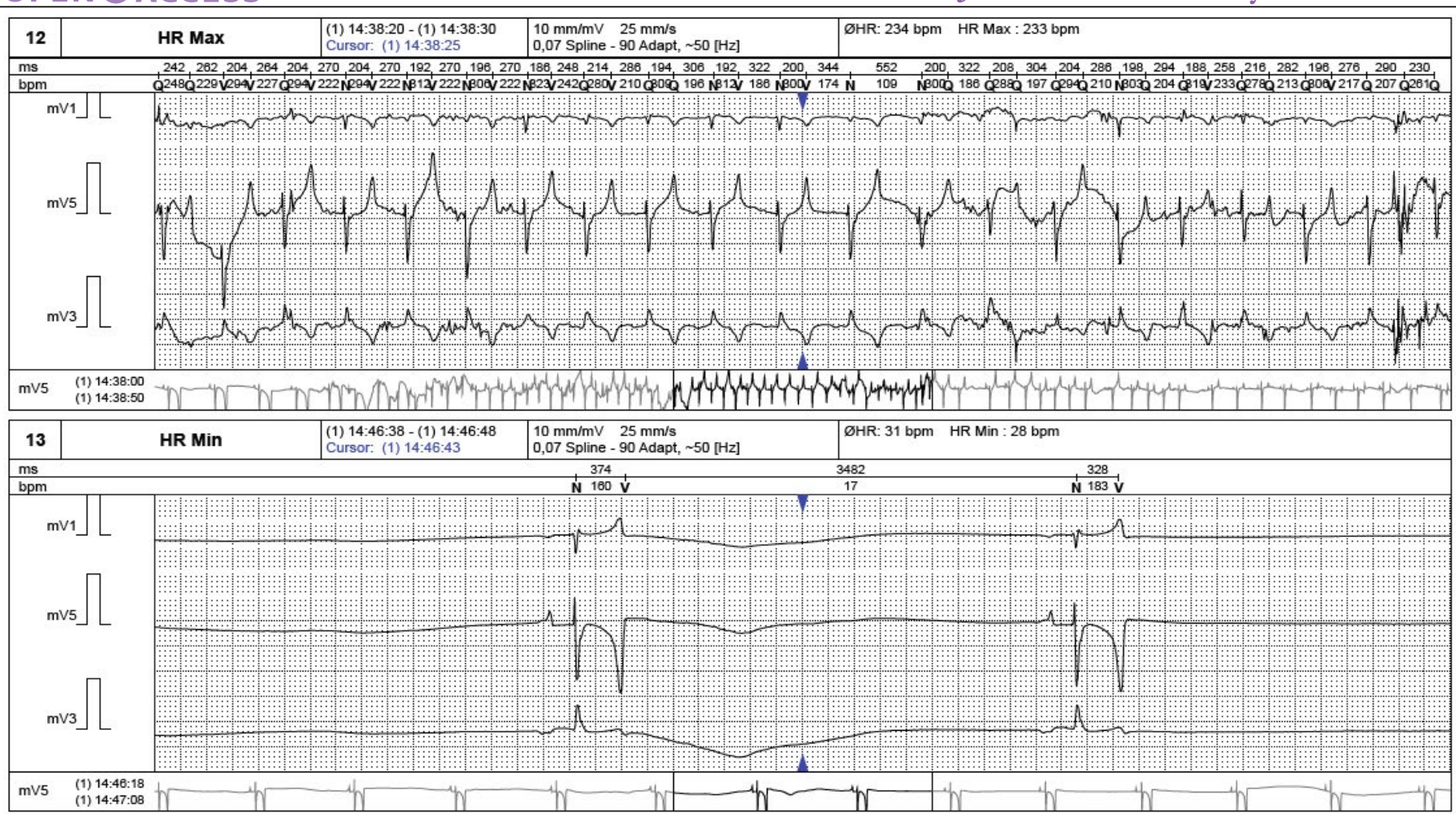

Figure 1: A section of Base-Apex lead ECG signal obtained from holter ECG recording.

tients with cardiovascular conditions (Vanderli et al., 2009). HRV contained time and frequency domain, the time domain parameters measuredfrom the peak of N-N intervals or R-R peak of the ECG which can be calculated as SDNN (standard deviation of the N-N intervals). Frequency domain or spectral analysis are measured by transforming ECG into a spectral signal. Previous study showed that the progression of cardiac conditions can be altered cardiac autonomic activity which leading to increase in heart rate and decrease in HRV (Pomeranz et al., 1985; Kishi et al., 2011). However, according to various usages of anesthetic agents, no studies have been performed to analyze the cardiac autonomic activity using HRV to desire anesthetic agents that provided the minimal cardiovascular effects in horses. The objective of this study was to evaluate the effects of various anesthetic agents on cardiac autonomic nervous activity using HRV analysis in horses and to provide the information of the anesthetic protocols for clinical application. We hypothesized that effects on cardiovascular, respiratory and recovery behavior of anesthetic agents in horse can be characterized using variables such as heart rate variability, respiratory and blood gas analysis.

\section{MATERIALS AND METHODS}

\section{Animals}

Eight Thai native crossbred gelding horses aged $6.1 \pm 0.9$ years, weighing $231.9 \pm 9.8 \mathrm{~kg}$ were used for this study. Horses in group of protocol 1 and protocol 2 had an average age of $5.50 \pm 1.5$ and $6.75 \pm 1.0$ and average weight was $223.7 \pm 13.7$ and $240.0 \pm 14.7$, respectively. The study conformed to the guidelines for Ethical Committee for Animal Experiments, Kasetsart University, Thailand. All horses were healthy bases on the clinical examinations. Horses were randomly assigned into 2 groups of 2 anesthetic protocols. Horses in group of protocol 1 were administered intravenous premedication and induction with acepromazine $(0.04 \mathrm{mg} / \mathrm{kg})+$ xylazine $(1 \mathrm{mg} / \mathrm{kg})+$ ketamine $(2.2 \mathrm{mg} / \mathrm{kg})$. Horses in group of protocol 2 underwent anesthesia using detomidine $(5 \mathrm{mcg} / \mathrm{kg})$ and ketamine $(2.2 \mathrm{mg} / \mathrm{kg})$. Then all horses were maintained under anesthesia for approximately 1 hour with a continuous infusion of $2 \%$ isofurane with an oxygen flow rate of $2 \mathrm{~L} / \mathrm{min}$. Horses were recovered in a quiet room with the padded stall. All parameters were recorded continuously for further analysis usinga blinded assessment.

\section{Cardiopulmonary Evaluations}

Routine laboratory evaluations were analyzed included hematology and serum biochemistry profiles as shown in Table 1. Electrocardiogram was recordedto evaluate heart rate and heart rate variability (Figure 1). Echocardiography was performed to rule out the cardiac pathology and to evaluate the heart contractility functions. Echocardiographic images were captured and stored for offline analysis. Left ventricular wall structure and function were calculated by measuring the images from two-dimensional plane (Figure 2). After anesthetic drugs administration, horses were intubated and positioned in right lateral recumbency. Arterial blood samples were collected from the facial artery 
OPEN OACCESS

and blood gas analysis were determined immediately using blood gas analyzer (Stat Profile pHOxplus, Nova Biomedical, United Kingdom).

Table 1: Blood profiles in 2 protocols

\begin{tabular}{|c|c|c|c|}
\hline & Protocol 1 & Protocol 2 & $\begin{array}{l}\text { Reference } \\
\text { range }\end{array}$ \\
\hline WBC (x103/ul) & $9.1 \pm 0.8$ & $8.3 \pm 0.7$ & $6-17$ \\
\hline $\mathrm{RBC}\left(\mathrm{x} 10^{6} / \mathrm{ul}\right)$ & $6.7 \pm 0.3$ & $7.7 \pm 0.3$ & $5-9$ \\
\hline HGB (g/dL) & $10.1 \pm 0.2$ & $12.5 \pm 0.4$ & $12-18$ \\
\hline $\operatorname{HCT}(\%)$ & $26.9 \pm 0.7$ & $31.3 \pm 0.8$ & $30-45$ \\
\hline PLT (x103/ul) & $276.5 \pm 37.3$ & $235.0 \pm 10.9$ & $200-900$ \\
\hline PROT (g/dL) & $6.6 \pm 0.2$ & $6.4 \pm 0.1$ & $5.3-7.8$ \\
\hline BUN (mg/dL) & $19.1 \pm 2.04$ & $15.9 \pm 1.5$ & $8-27$ \\
\hline Creatinine (mg/dL) & $1.25 \pm 0.1$ & $1.3 \pm 0.06$ & $<1.8$ \\
\hline $\operatorname{ALT}(\mathrm{U} / \mathrm{L})$ & $5.75 \pm 1.4$ & $5.0 \pm 1.9$ & $3-25$ \\
\hline AST (U/L) & $306.5 \pm 32.4$ & $319.3 \pm 16.7$ & $205-555$ \\
\hline ALP (U/L) & $183.8 \pm 19.5$ & $198.3 \pm 31.4$ & $109-315$ \\
\hline $\mathrm{CK}(\mathrm{U} / \mathrm{L})$ & $138.5 \pm 19.7$ & $110.3 \pm 15.8$ & $<200$ \\
\hline $\begin{array}{l}\text { Glucose }(\mathrm{mg} / \mathrm{dL}) \\
\text { Data represented as } \\
\text { RBC = Red blood cell } \\
\text { PLT = Platelet; PRC } \\
\text { nitrogen; ALT = Ala } \\
\text { aminotransferase; AI } \\
\text { kinase. (Reference ran }\end{array}$ & $\begin{array}{l}100.0 \pm 1.8 \\
\text { nean } \pm \text { SEM, } \\
H G B=\text { hemo } \\
\Gamma=\text { Total pro }\end{array}$ & $\begin{array}{l}96.5 \pm 3.2 \\
\text { VBC = Whi } \\
\text { globin; HCT } \\
\text { tein; BUN }\end{array}$ & $\begin{array}{l}60-130 \\
\text { e blood cell; } \\
=\text { hematocrit; } \\
\text { Blood urea } \\
=\text { Aspartate } \\
\mathrm{K}=\text { Creatine }\end{array}$ \\
\hline
\end{tabular}

\section{HRV Measurements}

Non invasive cardiac autonomic nervous control measurements were performed on 8 anesthetized horses using a holter ECG recording device (BTL medical technologies, Thailand). Three electrodes were placed on the shaved skin of the thorax with elastic tape to provide the 20 minutes
ECGs recording for the baseline and 60 minutes after administered anesthetic drugs. The recorded ECGs from every 10 minute sections were averaged and analyzed with analyzing program (BTL medical technologies, Thailand). Heart rate variability (HRV) was analyzed for the time domain and the frequency domain, time domain parameters were represented as the standard deviation of the $R R$ interval or SDNN and the frequency domain parameters were expressedas the activity in the high frequency $(0.15-$ $0.5 \mathrm{~Hz})$ and low frequency $(0.04-0.15 \mathrm{~Hz})$.
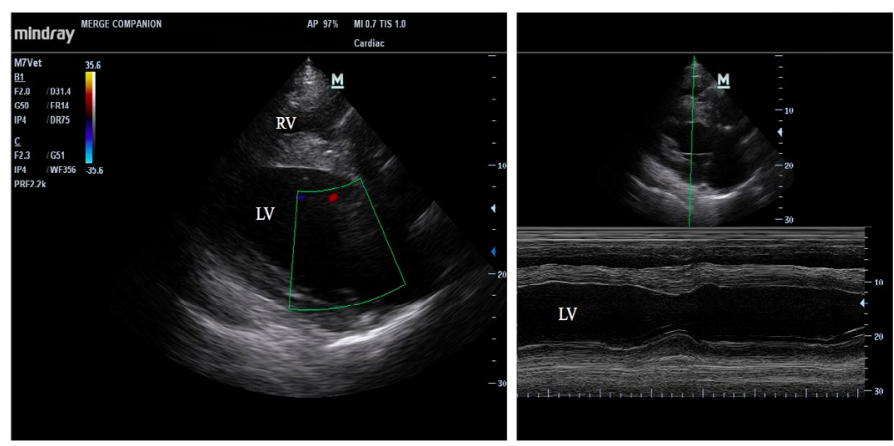

Figure 2: Echocardiographic of longitudinal image of heart (left) and $\mathrm{M}$ mode image of the left ventricle (right); $R V=$ right ventricle, and $L V=l$ eft ventricle

\section{Statistical Analysis}

All data are showed as mean \pm standard error of the mean (mean \pm SEM). Statistical analysis was performed using paired $t$-test and one-way ANOVA (Graphadprism software version 5). A $P$-value of 0.05 or less was indicated for statistical significance.

Table 2: Echocardiagraphic parameters in 2 protocols

\begin{tabular}{|c|c|c|c|c|c|c|}
\hline & Protocol 1 & & & Protocol 2 & & \\
\hline Parameters & Baseline & Post $30 \mathrm{~min}$ & Post $60 \mathrm{~min}$ & Baseline & Post $30 \mathrm{~min}$ & Post $60 \mathrm{~min}$ \\
\hline IVSd $(\mathrm{cm})$ & $2.51 \pm 0.2$ & $2.77 \pm 0.4$ & $2.86 \pm 0.4$ & $2.97 \pm 0.2$ & $2.87 \pm 0.2$ & $2.76 \pm 0.1$ \\
\hline LVIDd (cm) & $6.62 \pm 0.5$ & $7.17 \pm 0.6$ & $7.07 \pm 0.3$ & $8.69 \pm 0.6$ & $7.77 \pm 1.1$ & $8.18 \pm 0.7$ \\
\hline LVPWd (cm) & $3.01 \pm 0.3$ & $2.80 \pm 0.2$ & $2.92 \pm 0.2$ & $3.12 \pm 0.2$ & $2.85 \pm 0.1$ & $2.78 \pm 0.1$ \\
\hline IVSs (cm) & $3.33 \pm 0.2$ & $3.33 \pm 0.4$ & $3.40 \pm 0.3$ & $3.66 \pm 0.1$ & $3.04 \pm 0.1$ & $3.41 \pm 0.1$ \\
\hline LVIDs (cm) & $4.18 \pm 0.6$ & $4.90 \pm 0.5$ & $4.51 \pm 0.1$ & $5.85 \pm 0.6$ & $5.29 \pm 0.6$ & $5.41 \pm 0.5$ \\
\hline LVPWs $(\mathrm{cm})$ & $3.49 \pm 0.3$ & $3.23 \pm 0.3$ & $3.33 \pm 0.3$ & $3.66 \pm 0.2$ & $3.12 \pm 0.1$ & $3.15 \pm 0.1$ \\
\hline $\mathrm{EF}(\%)$ & $66.58 \pm 5.0$ & $56.0 \pm 4.3$ & $62.80 \pm 5.2$ & $55.38 \pm 4.9$ & $56.25 \pm 4.8$ & $60.17 \pm 2.3$ \\
\hline FS (\%) & $38.63 \pm 4.2$ & $31.50 \pm 2.6$ & $35.60 \pm 4.0$ & $30.56 \pm 3.6$ & $30.75 \pm 3.2$ & $33.7 \pm 1.7$ \\
\hline $\mathrm{SV}(\mathrm{mL})$ & $151.83 \pm 20.2$ & $160.04 \pm 26.8$ & $170.08 \pm 31.09$ & $241.80 \pm 42.8$ & $207.15 \pm 62.7$ & $223.8 \pm 46.7$ \\
\hline $\mathrm{CO}(\mathrm{L} / \mathrm{min})$ & $12.83 \pm 1.3$ & $14.13 \pm 3.1$ & $17.79 \pm 3.6$ & $17.26 \pm 2.2$ & $15.71 \pm 4.4$ & $19.2 \pm 4.0$ \\
\hline
\end{tabular}

Data represented as mean \pm SEM, IVSd; diastolic interventricular septum thickness, IVSs; systolic interventricular septum thickness, LVIDd; left ventricular end diastolic diameter, LVIDs; left ventricular end systolic diameter, LVPWd; left ventricular wall diastolic thickness, LVPWs; left ventricular wall systolic thickness. EF; The left ventricular ejection fraction, FS; Fractional shortening, SV; stroke volume, $\mathrm{CO}$; cardiac output. 
Table 3: The respiratory and hematological parameters in 2 groups of protocols

$\begin{array}{lllll}\text { Parameters } & \text { Protocol1 } & & \text { Protocol2 } & \\ & \text { baseline } & \text { After } 60 \text { mins } & \text { baseline } & \text { After } 60 \text { mins } \\ \text { Respiratory rate (beats/min) } & 19.8 \pm 3.0^{* * *} & 6.5 \pm 1.5^{* * *} & 25.0 \pm 2.8^{* * *} & 8.0 \pm 1.7^{* * *} \\ \mathrm{PaO}_{2} & 39.0 \pm 1.4^{* * * *} & 82.25 \pm 6.9^{* * * *} & 34.7 \pm 1.7 & 54.8 \pm 12.8 \\ \mathrm{PaCO}_{2} & 45.8 \pm 1.5^{* * * * *} & 91.8 \pm 1.7^{* * * * *} & 47.3 \pm 0.9^{* * *} & 76.6 \pm 6.2^{* * *} \\ \mathrm{pH} & 7.5 \pm 0.01 & 7.3 \pm 0.01 & 7.5 \pm 0.02 & 7.4 \pm 0.02 \\ \mathrm{HCO}_{3} & 35.9 \pm 1.0^{* * * *} & 45.7 \pm 1.0^{* * * *} & 37.1 \pm 0.6^{* * *} & 44.0 \pm 1.2^{* * *}\end{array}$

Data represented as mean $\pm \mathrm{SEM}, \mathrm{RR}=$ respiratory rate, $\mathrm{HR}=$ heart rate, $\mathrm{SDNN}=$ standard deviation of the $\mathrm{N}-\mathrm{N}$ intervals, $\mathrm{LF} / \mathrm{HF}=$ low frequency per high frequency ratio, $\mathrm{SBP}=$ systolic blood pressure, $\mathrm{DBP}=$ diastolic blood pressure, $\mathrm{MAP}=$ mean blood pressure, ${ }^{* *} P<0.01$ compared with baseline, ${ }^{* * *} P<0.001$ compared with baseline, ${ }^{* * * *} P<0.0001$ compared with baseline.

Table 4: HR, HRV, SBP, DBP and MAP parameters in 2 groups of protocols

\begin{tabular}{lllll} 
Parameters & $\begin{array}{l}\text { Protocol1 } \\
\text { baseline }\end{array}$ & After 60 mins & $\begin{array}{l}\text { Protocol2 } \\
\text { baseline }\end{array}$ & After 60 mins \\
\hline Heart rate (beats/min) & $74.5 \pm 2.8$ & $94.5 \pm 7.8$ & $89.5 \pm 9.9$ & $97.5 \pm 10.7$ \\
\hline SDNN & $381.0 \pm 57.2$ & $361.25 \pm 12.5$ & $318.0 \pm 14.4$ & $310.75 \pm 28.6$ \\
LF & $0.73 \pm 0.3$ & $0.76 \pm 0.3$ & $0.95 \pm 0.5$ & $0.78 \pm 0.3$ \\
HF & $0.88 \pm 0.5$ & $0.87 \pm 0.3$ & $0.97 \pm 0.6$ & $0.80 \pm 0.2$ \\
LF/HF & $0.90 \pm 0.05$ & $0.90 \pm 0.08$ & $0.77 \pm 0.07$ & $0.89 \pm 0.2$ \\
SBP & $116.3 \pm 17.7$ & $113.5 \pm 7.6$ & $105.3 \pm 16.9$ & $144.25 \pm 20.5$ \\
DBP & $66.3 \pm 2.8$ & $61.5 \pm 4.1$ & $69.3 \pm 10.1$ & $94.25 \pm 14.2$ \\
MBP & $82.0 \pm 5.1$ & $83.0 \pm 5.0$ & $80.8 \pm 12.3$ & $117.5 \pm 13.9^{*}$
\end{tabular}

Data represented as mean $\pm \mathrm{SEM}, \mathrm{HRV}=$ heart rate variability, $\mathrm{HR}=$ heart rate, $\mathrm{SDNN}=$ standard deviation of the $\mathrm{N}-\mathrm{N}$ intervals, $\mathrm{LF}=$ low frequency, $\mathrm{HF}=$ high frequency, $\mathrm{LF} / \mathrm{HF}=$ low frequency per high frequency ratio, $\mathrm{MBP}=$ mean blood pressure, ${ }^{*} P=0.09$ compared with baseline.

\section{RESULTS AND DISCUSSION}

All 8 horses (aged $6.1 \pm 0.9$ years and weighing $231.9 \pm 9.8 \mathrm{~kg}$ ) completed the study without any complications. There were no statistically different between groups concerning mean age and body weight $(p=0.52$ and $p=0.45$, respectively). Results of blood profiles and cardiac functions before the procedure are summarized in Table 1 and Table 2, respectively. Blood profiles and cardiac output in both groups were within the normal range, only minimal changes in echocardiography parameters were observed in this present study and there were no significant differences between the groups as determined by one-way ANOVA. Although the capacity of the blood pumped from ventricle per beat or stroke volume showed no significant differences between the groups but the baseline stroke volume in group of protocol 2 was higher than horse in group of protocol 1 . There was a possibility that the stroke volume would arise from the individual high in blood calcium level and myocyte contractility of one horse in the group of protocol 2.

All horses showed a degree of hypoventilation, overall respiratory rate decreased from a baseline value of $22.0 \pm 2.1$ bpm to $7.25 \pm 1.1 \mathrm{bpm}$. Horse showed a similar respiratory pattern and no apnea was observed during the anesthet- ic protocols. The increased in $\mathrm{PCO}_{2}$ was accompanied by the significantly increased in $\mathrm{HCO}_{3}$ in group of protocol 1 . Furthermore, $\mathrm{pH}$ also decreased and $\mathrm{PaO}_{2}$ significantly increased in this group when compared with baseline (Table 3).The results suggested to provide the ventilator support (high flow oxygen) during recovery when using these anesthetic protocols.

Heart rate and mean blood pressure (MBP) increased after anesthetic drug administered in both groups.Horses in group of protocol 1 had the higher percentage change of heart rate values $(26.8 \%$ vs $8.9 \%)$. MBP were increased in both protocols and slightly increased in group of protocol 2 ( $p=0.09$ as showed in Table 4 ). As far as the hemodynamic profiles were concerned, there was an increase in MBP in both groups as compared to anesthetic drug used in other studies. The increase in heart rate, $\mathrm{MBP}$ and $\mathrm{PaCO}_{2}$ may have influence from xylazine as previously reported (Muir et al., 1977; Meyer et al., 1985; Yamashita et al., 2000). The cardio respiratory profiles of this study was almost similar to many studies except that of Yamashita et al.2000, which showed the cardiac depression after intravenous administered xylazine or detomidine. This difference can be attributed to the combination of the anesthetic drugs to reduce the dose of detomidine. Other study used a higher dose of 
detomidine $40 \mathrm{mcg} / \mathrm{kg}$ as compared to the use of $5 \mathrm{mcg} /$ $\mathrm{kg}$ in this study.

Standard deviation of the NN interval (SDNN) decreased after anesthetic drug administration in both group of protocol (Table 4) indicated a reduction of parasympathetic tone which related to the slightly increase of the heart rate. The decreased of LF power were observed in both groups which indicated a vagal inhibition. The results from this study showed that anesthetic drugs in both protocols produced an increase in heart rate and had effect on the parasympathetic activity. The higher decrease of HF power was in group of protocol 2 indicated a high parasympathetic activity and horses in this group also had high values of the $\mathrm{LF} / \mathrm{HF}$ ratio when compared with another protocol.

Table 5: Recovery responses in 2 groups of protocols

\begin{tabular}{|lll|}
\hline First move (min) & Protocol 1 & Protocol 2 \\
\hline Head lift (min) & $4 \pm 0.7$ & $3.25 \pm 0.9$ \\
\hline First try sternal (min) & $5.25 \pm 0.5$ & $6.25 \pm 2.4$ \\
\hline Sternal (min) & $6.25 \pm 1.0$ & $6.75 \pm 2.5$ \\
\hline Number attempt to sternal & $3.25 \pm 1.3$ & $3.75 \pm 1.5$ \\
\hline First try to stand (min) & $11.75 \pm 2.0$ & $7.5 \pm 2.3$ \\
\hline Standing (min) & $8.5 \pm 3.0$ & $10.25 \pm 1.3$ \\
\hline Number attempt to stand & $5.25 \pm 3.9$ & $4 \pm 1.6$ \\
\hline Recovery quality (score) & $3.75 \pm 0.6$ & $2.5 \pm 0.6$ \\
\hline
\end{tabular}

The results suggested that acepromazine $(0.04 \mathrm{mg} / \mathrm{kg})+$ xylazine $(1 \mathrm{mg} / \mathrm{kg})+$ ketamine $(2.2 \mathrm{mg} / \mathrm{kg})$ might provide the better preservation of cardiac autonomic activity balance than detomidine. However, there was no marked HRV results on frequency domain variation was observed in any protocols. The results indicated that the ability of ECG holter device to measure the frequency domain of the HRV in horses was not substantially affected by the choice of these 2 anesthetic protocols. However, the anesthetized horse was significantly influenced by the respiratory rate and blood gas components. All horses recovered from anesthesia in a recovery room, the first movement was noted at approximately $3.6 \pm 0.5$ minutes (Table 5 ). There was no statistically different between group of protocol 1 and protocol 2 related to the recovery behavior. The duration for horses to complete sitting, sternal recumbency and standing was shorter in group of protocol 2. However, the overall recovery quality scores were higher in group of protocol 1. There were no differences between the recovery events of the two anesthetic protocols. An insufficient number of horses in each groupcould affect the statistically analysis and this was the limitation of this study. Furthermore, only one set of ECG were recorded during 20 minutes, a recording at different period of surgical protocol may affect the results. HRV analysis in anesthetized dogs still requires further investigations to ensure validity for use in the future application.

\section{CONCLUSION}

The findings of this study showed that acepromazine $(0.04$ $\mathrm{mg} / \mathrm{kg})+$ xylazine $(1 \mathrm{mg} / \mathrm{kg})+$ ketamine $(2.2 \mathrm{mg} / \mathrm{kg}) \mathrm{can}$ be used as a premedication and intubation in horse which could produce less effect on cardiovascular function and higher recovery score than other protocol used. The results suggested acepromazine $(0.04 \mathrm{mg} / \mathrm{kg})+$ xylazine $(1 \mathrm{mg} /$ $\mathrm{kg})+$ ketamine $(2.2 \mathrm{mg} / \mathrm{kg})$ should be used as a premedicate and intubation to provide the cardiovascular stable and recovery quality in horses.

\section{ACKNOWLEDGEMENTS}

Authors are grateful to Merge Companion (Thailand) Co., Ltd. that provided the portable color Doppler ultrasound. This research did not receive any specific grant from funding agencies in the public, commercial, or not for profit sectors.

\section{CONFLICT OF INTEREST}

There is no conflict of interest.

\section{AUTHORS CONTRIBUTION}

Soontaree Petchdee: A principle investigator, designed and performed experiments, analysed data and wrote the paper.

Aree Laikul, Juthamas Leklub and Jutamat Rattanakunuprakarn: Performed the experiments.

\section{REFERENCES}

- Carregaro AB, Luna SP, Mataqueiro MI (2007). Effects of buprenorphine on nociception and spontaneous locomotor activity in horses. Am. J. Vet. Res. 68: 246-250. https://doi. org/10.2460/ajvr.68.3.246

- Jarrett MA, Bailey KM, Messenger KM, Prange T, Gaines B, Posner LP (2018). Recovery of horses from general anesthesia after induction with propofol and ketamine versus midazolam and ketamine. JVAMA. 253: 101-107.

- Johnston JM, Eastment JK, Wood JLN (2002). The confidential enquiry into perioperative equine fatalities (CEPEF): mortality results of phase 1 and 2. Vet. Anaesth. Analg. 29: $159 \mathrm{e} 170$.

- Karcz M, Chojnowska L, Zareba W, Ruzytto W (2003). Prognostic significance of heart rate variability in dilated cardiomyopathy. Int. J. Cardiol. 87: 75-81. https://doi. org/10.1016/S0167-5273(02)00207-3

- Kato T, Ohmura H, Hiraga A, Wada S, Kuwahara M, Tsubone $\mathrm{H}$ (2003). Changes in heart rate variability in horses during immersion in warm springwater. Am. J. Vet. Res. 64: 1482- 
1485. https://doi.org/10.2460/ajvr.2003.64.1482

- Kishi T (2012). Heart failure as an autonomic nervous system dysfunction. J. Cardiol. 59:117-122. https://doi. org/10.1016/j.jjcc.2011.12.006

- Kishi T, Hirooka Y, Sunagawa K (2011). Auto implantation of astrocytes into the cardiovascular center of brain stem causes sympathoinhibition and decreases the mortality rate inmyocardial infarction-induced heart failure. Circulation. 124: A11489.

- Love EJ, Taylor PM, Whay HR (2013).Postcastrationanalgesia in ponies using buprenorphine hydrochloride. Vet. Rec. 172: 635-639. https://doi.org/10.1136/vr.101440

- Marly C, Bettschart-Wolfensberger R, Nussbaumer P (2014). Evaluation of a romifidine constant rateinfusion protocol with or without butorphanol fordentistry and ophthalmologic procedures in standinghorses. Vet. Anaesth. Analg. 41, 491e497. https://doi.org/10.1111/vaa.12174

- Meyer RE, Short CE (1985). Arterial to end-tidal $\mathrm{CO}_{2}$ tension and alveolar dead space in halothane or isoflurane anesthetized ponies. Am. J. Vet. Res. 46: 597-599.

- Muir WW, Skarda RT, Milne DW (1977). Evaluation of xylazine and ketamine hydrochloride for anesthesia in horses. Am. J. Vet. Res. 38: 195-201.

- Oliveira FA, Pignaton WA, Teixeira-Neto FJ (2014). Antinociceptive and behavioural effects of methadonealone or in combination with detomidine in conscious horses. J. Equine. Vet. Sci. 34: 380e386.

- Pagani M, Lombardi F, Guzzetti S, Rimoldi O, Furlan R, Pizzinelli P, Sandrone G, Malfatto G, Dell'Orto S, Piccaluga E (1986). Power spectral analysis of heart rate and arterial pressure variabilities as a marker of sympatho-vagal interaction in man and conscious dog. Circ. Res. 59: 178193. https://doi.org/10.1161/01.RES.59.2.178

- Pomeranz B, Macaulay RJ, Caudill MA, Kutz I, Adam D, Gordon D, Kilborn KM, Barger AC, Shannon DC, Cohen RJ (1985). Assessment of autonomic function in humans by heart rate spectral analysis. Am. J. Physiol. 248: H151-H153.

- Satue K, Hernandez A, Munoz A (2012). Physiological Factors in the Interpretation of Equine Hematological Profile. In: Hematol. Sci. Pract. 573-596.

- Vanderlei LC, Pastre CM, Hoshi RA, Carvalho TD, Godoy MF (2009). Basic notions of heart rate variability and its clinical applicability. Rev. Bras. Cir. Cardiovasc. 24: 205-217 https://doi.org/10.1590/S0102-76382009000200018.

- Yamashita K, Tsubakishita S, Futaok S, Ueda I, Hamaguchi H, Seno T, Katoh S, Izumisawa Y, Kotani T, Muir WW (2000). Cardiovascular effects of medetomidine, detomidine, and xylazine inhorses. J. Vet. Med. Sci. 62: 1025-1032. https:// doi.org/10.1292/jvms.62.1025 